\title{
Intelligent Agent Architecture for Context Aware Service
}

\author{
Mechelle Grace Zaragoza ${ }^{1}$ and Haeng-Kon Kim ${ }^{2 *}$ \\ ${ }^{1,2}$ School of Information Technology, Catholic University of Daegu, Korea \\ ${ }^{1}$ mechellezaragoza@gmail.com, ${ }^{2}$ hangkon@cu.ac.kr
}

\begin{abstract}
A google search of smart agent keywords will return more than 330,000 hits; the multiple agent returns almost double this amount, as well as more than 5,000 appointments on www.citeseer.com. Tounderstand fully what agent technology is and what has led to its huge popularity in the academic and commercial world, agent-based system technology provides a new paradigm for the design and implementation of software systems. The ultimate goal of this study is to develop an intelligent agent platform for the ontological context-based service and apply the intelligent agent platform developed to the health care application domain.
\end{abstract}

Keywords-Artificila Intelligent, Intelligent Agent, Context Aware Service

\section{Introduction}

Agent architectures can be considered as agent software engineering models; Researchers in this field are primarily concerned with the problem of designing software or hardware systems that satisfy the properties specified by agent [1].

Since an AI system is composed of an agent and its environment, the environment may contain other agents. An agent is all that your environment can perceive through sensors and acts in this environment through effectors. A human agent has sensory organs, such as eyes, ears; nose, tongue, and skin are parallel to sensors and other organs, such as hands, legs, and mouth, for effectors. A robotic agent replaces cameras and infrared range finders such as sensors and several motors as well as actuators for effectors.

\subsection{A Software Agent Encoded Bitstreams as Programs and Actions}

Terminology of the agent:

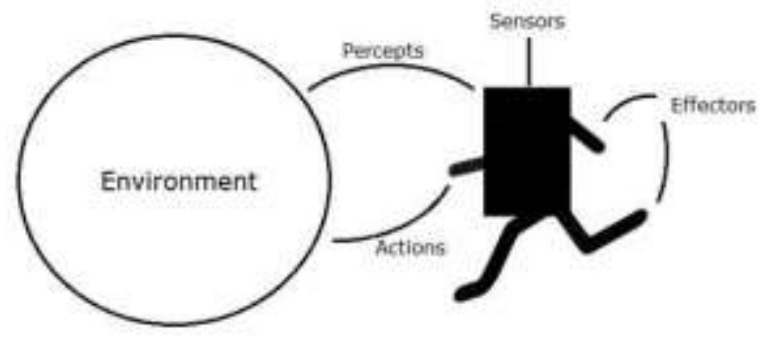

Figure 1. Agent and Environment

- Agent Performance Measure: Is the criterion that determines the success of an agent.

- Behavior of the agent: This is the action that the agent performs after a given sequence of perceptions.

Received (January 16, 2018), Review Result (March 24, 2018), Accepted (March 28, 2018)

* Corresponding Author 
- $\quad$ Perception - These are the perceptual entries of the agent in a given instance.

- Percept Sequence - This is the story of everything an agent has seen so far.

- Agent function: is a card from the sequence of the precept to an action [2].

\subsection{Context Aware}

Context is defined as the interrelated conditions in which something exists or occurs. While this is a general definition, it does not help much for understand the concept in a computer environment. The use of the word "context" tends to be vague because everything in the world happens in a certain context. The term has been used in many ways in different fields of computer science, such as "contextual help", "contextual search", "multitasking", "change of context", "psychological context perception", and so on We focus only on the context used by applications in mobile computing [3].

To derive the context of the knowledge service through efficient contextual reasoning, this study proposes a model of consciousness in the context of semantic technology based on ontologies, an intelligent example of construction of the KIN textual knowledge base. In addition, the developed intelligence agent platform will be used to build a contextual information system based on optimized cullology for real-world applications. Location Cognition is one of the main ubiquitous computing technologies receiving various data from different types of devices and understanding and addressing the user's location information to provide the most appropriate current situation.Contextual awareness means that we can use the context information. A system is aware of the context if it can retrieve, interpret and use the contextual information and adapt its features to current context that is being used. The term content computing is generally understood by those who work in aware of the context, where the context is considered the key to their efforts to disperse and weave computer technology seamlessly. One of the goals of context-based systems is to acquire and use information about the context of a device to provide services which are appropriate for the people, place, time, and the event [4].

There was a period of initial activity in the conscious contextual context; however, the activity seems to increase drastically. Nowadays, to overcome new challenges and requirements found in context-sensitive systems, many researchers have made efforts to design and implement the network, the user infrastructure and middleware that efficiently deliver contextaware users services. In other words, many people are interested in context-aware systems. Therefore, we believe this is a good time for a review and analysis. Currently, it is difficult to compare articles because the research is published in very different journals. Therefore, the main objectives of this review are: Classify and summarize relevant research for the conscious context systems and to provide a conceptual framework for integration and classification of such topics.

To understand this concept, an example of automatic light as a context sensitive system was discussed in the study. In the entrances to houses and hotel corridors, automatic lights have become commonplace. These systems can also be considered as simple contextsensitive systems. The contextual parameters taken into account are the current lighting conditions and if there is a movement nearby. The coping mechanism is quite simple. If the situation detected is dark and movement is detected, the light turns on. The light turns on while the person is moving and after a period of time during which movement is not detected, the light goes off again. Likewise, the light will go off if there is the presence of light. This simple example describes the basic principle of a context aware system. Reference architecture for context-aware computer systems is shown.

Sensors provide data on real-world activities and events. Perceptual algorithms will give meaning to these stimuli and classify situations in their context. Depending on the context, the actions of the system will be activated [5]. 


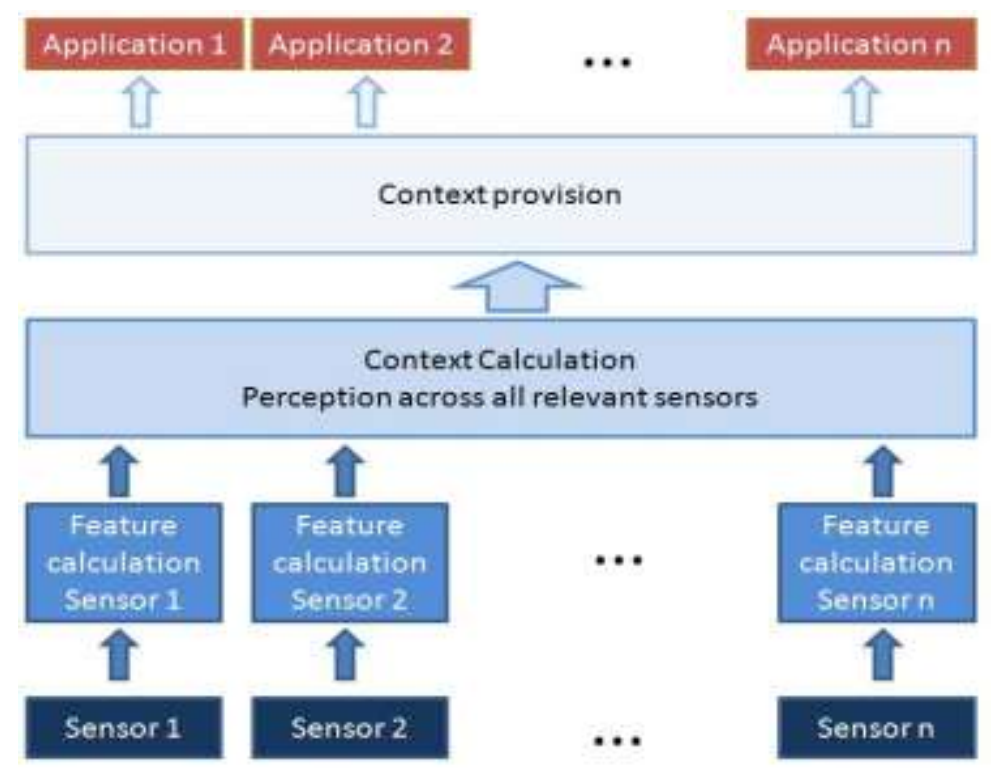

Figure 1. Depicts Reference Architecture for Context-Aware Computing Systems

\subsection{Ontology}

Ontology has many definitions, of which Thomas Gruber is the most famous. Gruber defines ontology as a formal () and clear () specification that shares the concept of 0 (0), which is a recognized knowledge of the shared "O". (Or vocabulary from the unit) which is accepted by the consent of all members participating in the region, rather than individuals. The "conceptualization 0 " is an abstract model. "Formal" means that the computer must be able to understand the content of the ontology. Finally, "a clear zero means that we must define the conditions and restrictions that apply when modeling a particular area.

The ontology can be divided into topology and background ontology according to the field of application. The ontological topology does not depend on a specific domain because it focuses on basic and universal concepts that can be applied in various fields. Therefore, higher ontology is mainly used to represent the real world by modeling common sense concepts. The scope of the ontology is not limited to a domain. If there are universal ideas common to many concepts in any field, the domain that includes these concepts can also be considered as a parent ontology. In other words, this means that there may be a higher ontology in which general concepts are collected even in a domain, and there are different superior ontologies for contextual systems in the context. One of the characteristics of ontology, reuse and ontology, one of the hallmarks of dog use and inheritance, and the existing ontology can be used together to build more ontologies. Ontology based on semantic web can use a common format objectively defined and give meaning to the information so that the system can know an effective situation by sharing, understanding and reasoning of the situation information. That is, the user can provide a service adapted to a given situation.

First, there is an increase in expressiveness. The ontology language uses an objectbased modeling approach. As a result, it acquires the characteristics of several types of contextual information and has a sufficient representation capacity to express it.

The second is that the exchange of knowledge of the situation is possible. The use of ontology to represent contextual information can have a common concept and meaning in the interaction process of several entities in a context-aware computing environment. The third is the capacity for reasoning. Ontology-based context recognition systems can use a 
variety of logic-based reasoning mechanisms. We can derive new situation information using the existing situation information, and the resulting situation information helps to provide various services, such as learning and referral. The fourth is the reuse of knowledge.

By reusing well-defined ontologies from different domains, it is possible to easily and efficiently build large-scale context ontology without paying a lot of money and scalability. The concept of ontology is generally composed of a hierarchy of terms. As a result, new contextual ontologies can be added and expanded using hierarchical methods.It is essential to abstract the concept of the real world into a unified and specific model according to the machine-readable standard. Among the different types of modeling techniques that are currently available, the method corresponding to the previous one is the ontological model. The corridor consists of a knowledge base that stores the reimbursement and knowledge, a reasoning engine, a situation acquisition module and a security management module.

The contextual knowledge base summarizes and models the contextual information and manages the metadata that can be derived based on the instantiated data and the real information. The contextual reasoning engine plays the role of inferring new knowledge and other situations with acquired situational information and finding and resolving incompatible knowledge. The situation acquisition module collects data from all sources that can be obtained from various broadcasting organizations, agents and situations distributed in space, and processes them so that they can be used as input for an inference engine or a situation management module. Finally, the security management module manages the policies to share and control the user's personal information, and applies them to deduce the situation or deduct knowledge.

The situational context knowledge service system comprises of certain layers. It is an application that receives context information from several intelligent agents and provides information to the user receives the data from the server and shows it to the user. Again, context recognition devices are classified as information providers and external providers. (Internal), public information such as weather information, traffic jams and local information must be retrieved from other external institutions, and contextual servers must be able to handle these various situations. This must be able to receive cognitive information. Use it to process the server in different types of information in the server's data. There is a sensor adapter for this function, and the sensor adapter stores the processed data and forwards it to the system. We need a knowledge base for reasoning (based on ontology) and a module for security as much as we deal with various data inconsistencies. We also need to build a database of data sensors to store other reference data.

The inference engine makes inferences based on these various data, and the proper management transfers the data to the broker. The broker interprets the received data and passes the data of the beam to the corresponding user's device. The user's application receives this result data and provides it to the user. In order to build a context aware system, researches about user application, information processing server, and context aware devices will be involved. However, the most important part is the context server part, which will be built on an intelligent agent basis. This part will include an inference engine, a knowledge base, and a privacy module. We will also examine some of the top ontologies already provided by various organizations in order to construct the ontology for reasoning.

\section{Related Literature}

On the internet and mobile devices, Zhang has opened the era of the new evolution of the computer, and several devices and platforms have been opened, and many services are offered to users. The omnipresent is no longer the story of the future because of the small 
computers that are available everywhere and the broad connection of internet. Nowadays, the concept of Thing Internet (IoT) and Everything Internet have come to embroider. Various devices detect the situation in real time through sensors or labels, and the devices are interconnected to exchange information, thus providing the right service for the user.

The situation awareness service is an intelligent and easy-to-use service provided by the use of several devices. In particular, it perceives the surrounding situation and the user's intention and creates the optimal service environment that responds to the situation. It builds the environment in an active and autonomous way without human intervention, it adapts according to the evolution of the situation and the habits of the user. Ubiquitous computing has been the subject of a series of investigations on computer technology.

The situation recognition system is based on a method of direct sensor use and a middleware infrastructure structure according to different methods of information acquisition from the client device ranging from various sensors to laptops. The architecture of the context awareness system based on the context server, the context awareness model to define and store contextual data in a machine-readable form, and in particular, the ubiquitous middleware is composed of situational knowledge. The middleware for reporting location information includes the sensor layer, the agent layer, the situation service layer and the application layer.

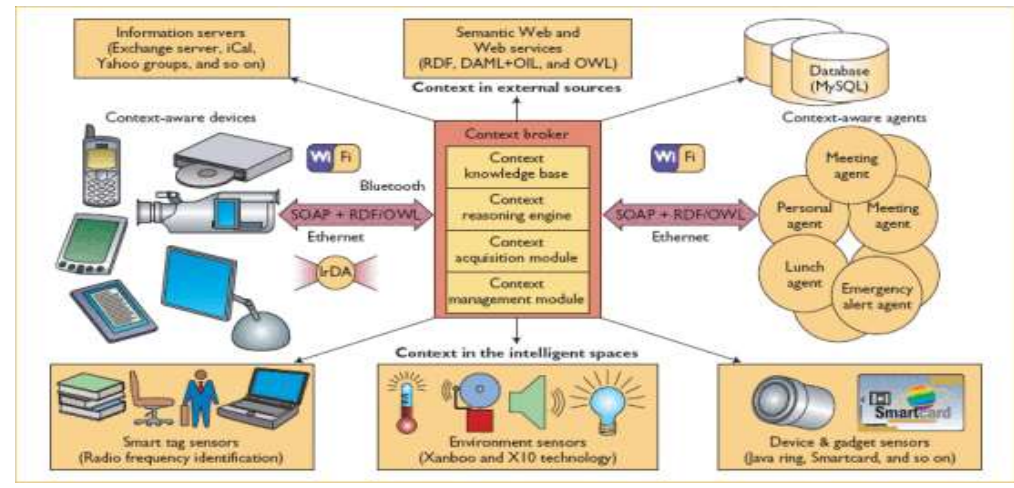

Figure 2. EasyMeeting Architecture

EasyMeeting is an extension of Vigil, a third-generation computing infrastructure developed at the University of Maryland, Baltimore County (UMBC). Although the development of research behind Vigil shows great promise in building flexible and safe, intelligent spaces, it lacks the necessary support for contextual awareness and privacy protection. To improve the previous system, the researcher added the contextual support to EasyMeeting by exploiting Cobra. Figure 2 illustrates the architecture of the EasyMeeting system.Context Broker Architecture $(\mathrm{CoBrA})$ is an agent-based architecture that supports context-aware systems in smart spaces (for example, smart meeting rooms, smart homes, and smart vehicles). A central feature of this architecture is an intelligent agent called a context broker that maintains a shared context model on behalf of a community of agents, services, and devices in the space and provides privacy protections for users.

\subsection{The Main Differences between CoBrA and other Similar Architectures}

CoBrA uses Web Ontology Language OWL, a W3C semantic Web standard, to define context ontologies (people, agents, devices, events, time, space, etc.). In other systems, the context is often implemented as programming language objects (for example, Java classes), lacking expressive power to support context reasoning and high-level knowledge exchange. 
CoBrA provides a rich context agent to maintain a shared context model for all entities in an associated space. In other systems, it is usually required that the individual entities administer their own contextual knowledge.

CoBrA allows users to set a privacy policy to control the sharing and use of their situation information (for example, where they are, with whom they are, what they do). In other systems, IT entities are generally free to share any situational information acquired from a user. [6-7]

CORBA [Obj91] is a middleware for the independent development of the architecture of Distributed applications based on transparent objects for the programmer. Object request Intermediaries (ORBs) facilitate communication between heterogeneous nodesenvironments at the object level. They provide mechanisms for object discovery and creating instances in remote machines, and classifying and dematerializing the object. In addition, ORBs manage security, recovery of objects and method invocations. CORBA also provides a significant amount of support services. The Event services and notifications provide a substrate for facilitating asynchronous interactions between objects. The name service handles associations between names and objects, including the binding name and resolution. The collection service allows the manipulation of several objects in a group.

\subsection{Identify the Following Challenges for an Application Model for Ubiquitous Computing}

A. Neutral application of the device: an application must be developed independently of the device on which it can be used, to accommodate the variety different devices available for use in generalized environments.

B. High-level user interaction: For an application to be independent of the device,the description must capture the purpose of the user interaction at a high level, instead of including the rigid decomposition of the interaction, which can be specific to the device.

C. Abstract descriptions of services: in the dynamic resources of generalized environments appear and disappear with time. Therefore, an application model must not make assumptions about the availability of services, rather than they should be specified abstractly [8].

\subsection{Context Acquisition and Representation of RTE}

The lower level of the hierarchy is the context of layer detection provided by a set of hardware and software sensors that continuously probe the characteristics of the wireless network, temporal and spatial data, device characteristics and background and user preferences. The context detection layer generates quantized and unquantized raw data whose values are numeric values, boolean and literal values, and most of them are time stamped. To transform this context data into meaningful contextual information that can be used by the reasoning engine, the raw data is translated into symbolic information. Mapping is done through the context perception layer through computer techniques, inference and learning. The perception context of the layer is independent of the context of the detection technology in that it provides an abstract representation. At the top level, there is the Context Adaptation Layer, where learning services are discovered and the content of learning is adapted to the perceived context. The higher ontology describing the knowledge of all elements of the context increases with the subject domain ontology, and is used as a knowledge base for unified system reasoning. The result of the reasoning process is a set of extracting metadata used for discovery and adaptation services of the context-based learning system (e.g., the device framework and the environment) and the student-centered context ( that is, the student and the context of the activity). In particular, 
the extracted metadata is used to customize the learning path and learning content according to the student background, prerequisites, previous tasks, available network bandwidth, network security and other connectivity issues. Each of these adaptations is controlled by the logic of adaptation to context in the form of ontological reasoning [9].

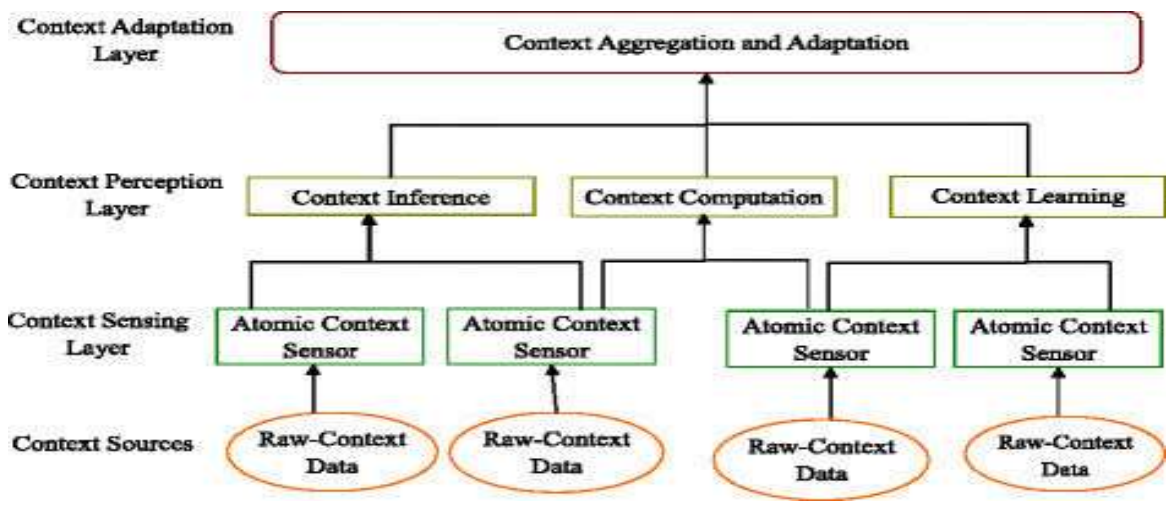

Figure 3.RTE Conceptual Model

\section{Development ofIntelligent Agent Platform for Ontology based Context Aware Service}

Gaia, who studies at the University of Illinois, provides operating system functions (event file system, security and process signals, etc.,) to space out physical space such as rooms, houses, buildings and airports. Gaia is a contextual object that defines an application, user, service, device, location, and activity.

To resolve the diversity and complexity of contextual awareness through the use of the model in the face, we apply the context-based awareness to collect situation information and allow the agent to take action using different machines. However, instead of woL's standard woL, the ontology was defined using its predecessor, DAML + OIL, and the processing of situation information based on first-order logic was sought to infer the context. Figure 4, shows the Gaia structure.

\begin{tabular}{|c|c|c|c|c|c|c|c|}
\hline \multirow{3}{*}{$\begin{array}{c}\text { Midd } \\
\text { lewar } \\
\text { e }\end{array}$} & \multicolumn{7}{|c|}{ Active SpaceApplications } \\
\hline & \multicolumn{3}{|c|}{ Application Framework } & \multicolumn{4}{|c|}{ Quality of Service } \\
\hline & $\begin{array}{l}\text { Context } \\
\text { Service }\end{array}$ & $\begin{array}{l}\text { Context } \\
\text { File } \\
\text { System }\end{array}$ & $\begin{array}{l}\text { Component } \\
\text { Repository }\end{array}$ & $\begin{array}{c}\text { Event } \\
\text { Manager }\end{array}$ & $\begin{array}{l}\text { Presence } \\
\text { Servicee }\end{array}$ & $\begin{array}{c}\text { Spare } \\
\text { Reposit } \\
\text { ory }\end{array}$ & $\begin{array}{l}\text { Security } \\
\text { Service }\end{array}$ \\
\hline & \multicolumn{7}{|c|}{ Component Management Core } \\
\hline
\end{tabular}

Figure 4. Ontology Ubiquitous and Pervasive Applications GAIA

In the semantic web of the UbiComp Special Interest Group, we propose the SOUPA (Standard Ontology for ubiquitous and generalized applications) and establish a unified upper ontology of several contexts that occur in ubiquitous environments and favor their 
use in ontological development. SOUPA is FOAF, DAML-TIME, OpenCyc, CoBrAONT and many other ontologies. SOUPA has the advantage of developing an ubiquitous ontology-based service, and can be developed easily and quickly regardless of the development environment. SOUPA is divided into central ontology and extension ontology and is expressed in ontology WOL (web ontology language). The central ontology defines the higher-level context terminology that can be used in the general domain, and the extension ontology includes not only the central ontology, but also a specific type of ontology by application.

It consists of three layers. The upper layer receives the service information frame from the context application context server and provides the necessary content to the user. As can be seen in related studies in Korea and abroad, the contextual awareness system is a computer system capable of providing services and gigabytes appropriate to normal users. Contextual modeling of the expression and storage of contextual information so that the machine can understand it. Second, contextual reasoning on how to understand contexts based on information obtained from the physical environment. In this paper, we propose an interoperability framework for the exchange of context knowledge between heterogeneous and distributed IT entities. However, most studies are limited to building a concrete knowledge base and presenting an abstract model without the "ontological design" inference rule. To solve this problem, the Semantic Web has similar conditions. Therefore, in order to derive an effective context knowledge service, this study builds context-based contextualization and knowledge-based knowledge using ontology based on semantic web technology [10-14].

\section{Conclusion}

Research Results and Application

- Robotics and health care sector will be developed and evolved in the aging society and future society. It is the business model that can develop the intelligent software based technology in this field.

- Creation of synergy effect

- Intelligent agent platform for context aware service based on ontology

- Applicable to contextual service model of environmental agriculture and livelihood

- When utilizing personalized healthcare service platform, The number of visits to the hospital and the cost of repeated examination can be saved, as the number of households, fitness clubs, and work areas are expanded to the area of everyday life (for example, diabetes, electrocardiogram, blood pressure management using mobile phones), and unnecessary people

- To Prevent health insurance spending In addition to storing and managing basic clinical images and drug data measured in hospitals through a dance-type healthcare service platform, health information that can be acquired personally, ie, food, sleep, emotion, By managing data on exercise in a comprehensive way,

- Provide the ability for modern people to support smart living by enabling individual health information management

- Patient-centered medical service platform can be used for personal health management requiring continuous and preventive disease management It is easy for individuals to manage their own health data through mobile devices, thereby contributing to the improvement of quality of life in an aging society. Intelligent context aware platform, application technology for smart robots has been developed as a parcel image recognition and context knowledge base for robot control. Improve work environment and reduce cost 


\section{Acknowledgment}

This Research was supported by the MSIP (Ministry of Science, ICT and Future Planning), Korea, under the ITRC (Information Technology Research Center) support program (IITP-2018-2013-1-0087) supervised by the IITP (Institute for Information \& Communication Technology Promotion).

Following are results of the study on the, "Leaders in Industry-University Cooperation + "Project, supported by the Ministry of Education and National Foundation Korea.

\section{References}

[1] M. Wooldridge and N. R. Jennings, "Intelligent agents: Theory and practice", The knowledge engineering review, vol. 10, no. 2, (1995), pp. 115-152.

[2] AI - Agents \& Environments, https://www.tutorialspoint.com/artificial_intelligence/artificial_ intelligence_agents_and_environments.htm.

[3] G. Chen and D. Kotz, "A survey of context-aware mobile computing research", Technical Report TR2000-381, Dept. of Computer Science, Dartmouth College, 2000.http://ieeexplore.ieee.org/document/1355924/, vol. 1, no. 2.1.

[4] J.-y. Hong, E.-h. Suh and S.-J. Kim, "Context-aware systems: A literature review and classification", Expert Systems with Applications, vol. 36, no. 4, (2009), pp. 8509-8522.

[5] A. Schmidt, "Context-Aware Computing", The Encyclopedia of Human-Computer Interaction, 2nd Ed, https://www.interaction-design.org.

[6] H. Chen, T. Finin, A. Joshi, L. Kagal, F. Perich and D. Chakraborty, "Intelligent agents meet the semantic web in smart spaces", IEEE Internet Computing, vol. 8, no. 6, (2004), pp. 69-79.

[7] H. Chen, T. Finin and A. Joshi, "An Intelligent Broker for Context-Aware Systems", Adjunct Proceedings of Ubicomp 2003, Seattle, Washington, USA, (2003) October 12-15.

[8] M. Vuković, "Context aware service composition (No. UCAM-CL-TR-700)", University of Cambridge, Computer Laboratory, (2007).

[9] R. Benlamri and X. Zhang, "Context-aware recommender for mobile learners", Human-centric Computing and Information Sciences, vol. 4, no. 1, (2014), 12.

[10] J. Y Choi. Y. S. Ko, G. Kang Lee and W. S. Choi, "Healthcare 3.0 A world of healthy life", CEo information, Samsung Economic Research Institute, vol. 831, (2011).

[11] K. T. Oh and J. Lee, "Smart Life revolutionary real and smartphone addiction", Internet and Information Security, vol. 3, no. 4, (2012).

[12] J. H. Park and T. Hwangbo, "Healthcare IT Convergence Technology", Journal of Korea Information and Communications, vol. 28, no. 5, (2011).

[13] J. Song and H. Kim Jung, "Protection of health information for u-healthcare service", Journal of Information Security, vol. 17, no. 1, (2007).

[14] S. H. Kim, "IT convergence technology trends and forecasts (mainly Health)", Journal of Electronics, vol. 37, no. 6, (2010). 
International Journal of Control and Automation

Vol. 11, No. 10 (2018) 